\title{
Commutators associated with Schrödinger operators on the nilpotent Lie group
}

\section{Tianzhen Ni and Yu Liu* (D)}

*Correspondence: liuyu75@pku.org.cn School of Mathematics and Physics, University of Science and Technology Beijing, Beijing, China

\begin{abstract}
Assume that $G$ is a nilpotent Lie group. Denote by $L=-\Delta+W$ the Schrödinger operator on $G$, where $\Delta$ is the sub-Laplacian, the nonnegative potential $W$ belongs to the reverse Hölder class $B_{q_{1}}$ for some $q_{1} \geq \frac{D}{2}$ and $D$ is the dimension at infinity of $G$. Let $\mathcal{R}=\nabla(-\Delta+W)^{-\frac{1}{2}}$ be the Riesz transform associated with $L$. In this paper we obtain some estimates for the commutator $[h, \mathcal{R}]$ for $h \in \operatorname{Lip}_{\nu}^{\theta}$, where Lip $p_{v}^{\theta}$ is a function space which is larger than the classical Lipschitz space.
\end{abstract}

MSC: 22E30; 42B35; 35J10; 42B20

Keywords: commutator; Lipschitz space; nilpotent Lie groups; reverse Hölder inequality; Riesz transform; Schrödinger operator

\section{Introduction}

Assume $G$ to be a connected and simply connected nilpotent Lie group and $\mathfrak{g}$ to be its Lie algebra identified with the space of left invariant vector fields. Given $X=\left\{X_{1}, \ldots, X_{l}\right\} \subseteq \mathfrak{g}$, a Hörmander system of left invariant vector fields on $G$. Let $\Delta=\sum_{i=1}^{l} X_{i}^{2}$ be the subLaplacian on $G$ associated with $X$ and the gradient operator $\nabla$ be denoted by $\nabla=$ $\left(X_{1}, \ldots, X_{l}\right)$. Following [1], one can define a left invariant metric $d$ associated with $X$ which is called the Carnot-Carathéodory metric: let $x, y \in G$, and

$$
d(x, y)=\inf \{\delta|\exists \gamma:[0, \delta] \rightarrow G| \gamma(0)=x, \gamma(\delta)=y\},
$$

where $\gamma$ is a piecewise smooth curve satisfying

$$
\gamma^{\prime}(s)=\sum_{i=1}^{l} a_{i}(s) X_{i}(\gamma(s)), \quad \forall s \in[0, \delta], \sum_{i=1}^{l}\left|a_{i}(s)\right|^{2} \leq 1 .
$$

If $x \in G$ and $r>0$, we will denote by $B(x, r)=\{y \in G \mid d(x, y)<r\}$ the metric balls. Assume $\mathrm{d} x$ to be the Haar measure on $G$. Then, for every measurable set $E \subseteq G,|E|$ denotes the measure of $E$. Suppose $e$ to be the unit element of $G$. Note that $V(r)=|B(e, r)|=|B(x, r)|$ for any $x \in G$ and $r>0$. It follows from in [2] or [3] that there exists a constant $C_{1}>0$ such

(c) The Author(s) 2017. This article is distributed under the terms of the Creative Commons Attribution 4.0 International License (http://creativecommons.org/licenses/by/4.0/), which permits unrestricted use, distribution, and reproduction in any medium, provided you give appropriate credit to the original author(s) and the source, provide a link to the Creative Commons license, and indicate if changes were made. 
that

$$
\begin{array}{ll}
C_{1}^{-1} r^{d} \leq V(r) \leq C_{1} r^{d}, & \forall 0 \leq r \leq 1 ; \\
C_{1}^{-1} r^{D} \leq V(r) \leq C_{1} r^{D}, & \forall 1 \leq r<\infty,
\end{array}
$$

where $d$ and $D$ denote the local dimension and the dimension at infinity of $G$, and there is $D \geq d>0$. At this time, the Lie group $G$ is also called a Lie group of polynomial growth. If $G$ is a stratified Lie group, then $D=d$ (cf. [1]). Also, there exist positive constants $C_{2}, C_{3}>1$ such that

$$
\begin{aligned}
& C_{2}^{-1}\left(\frac{R}{r}\right)^{d} \leq \frac{V(R)}{V(r)} \leq C_{2}\left(\frac{R}{r}\right)^{D}, \quad \forall 0<r<R<\infty ; \\
& V(2 r) \leq C_{3} V(r) .
\end{aligned}
$$

Throughout this paper, we always assume that $d \geq 2$.

Let $L=-\Delta+W$ be the Schrödinger operator, where $\Delta$ is the sub-Laplacian on $G$ and the nonnegative potential $W$ belongs to the reverse Hölder class $B_{q_{1}}$ for some $q_{1} \geq \frac{D}{2}$ and $D>3$. The Riesz transform $\mathcal{R}$ associated with the Schrödinger operator $L$ is defined by

$$
\mathcal{R}=\nabla(-\Delta+W)^{-\frac{1}{2}}
$$

Let $b$ be a locally integrable function on $G$ and $T$ be a linear operator. For a suitable function $f$, the commutator is defined by $[b, T] f=b T(f)-T(b f)$. Many researchers have paid attention to the commutator on $\mathbb{R}^{n}$. It is well known that Coifman, Rochberg and Weiss [4] proved that $[b, T]$ is a bounded operator on $L^{p}$ for $1<p<\infty$ if and only if $b \in \mathrm{BMO}\left(\mathbb{R}^{n}\right)$, when $T$ is a Calderón-Zygmund operator. Janson [5] proved that the commutator is bounded from $L^{p}\left(\mathbb{R}^{n}\right)$ into $L^{q}\left(\mathbb{R}^{n}\right)$ if and only if $b \in \operatorname{Lip}_{v}\left(\mathbb{R}^{n}\right)$ with $v=\left(\frac{1}{p}-\frac{1}{q}\right) n$, where $\operatorname{Lip}_{v}\left(\mathbb{R}^{n}\right)$ is the Lipschitz space. Sheng and Liu [6] proved the boundedness of the commutator $[b, \mathcal{R}]$ from the Hardy space $H_{L}^{p}\left(\mathbb{R}^{n}\right)$ into $L^{q}\left(\mathbb{R}^{n}\right)$ when $b$ belongs to a larger Lipschitz space. Comparatively, there has been much less research on the commutator on nilpotent Lie groups. The goal of this paper will be to obtain some estimates for the commutator related to the Schrödinger operator on nilpotent Lie groups. The complicated structure of nilpotent Lie group will bring some essential difficulties to our estimates in the following sections.

Note that a non-negative locally $L^{q}$ integrable function $W$ on $G$ is said to belong to $B_{q}$ $(1<q<\infty)$ if there exists $C>0$ such that the reverse Hölder inequality

$$
\left(\frac{1}{|B|} \int_{B} W(y)^{q} \mathrm{~d} y\right)^{\frac{1}{q}} \leq C\left(\frac{1}{|B|} \int_{B} W(y) \mathrm{d} y\right)
$$

holds for every ball $B$ in $G$.

We first introduce an auxiliary function as follows.

Definition 1 Let $W \in B_{q}$ for some $q \geq \frac{D}{2}$. For $x \in G$, the function $m(x, W)$ is defined by

$$
\frac{1}{m(x, W)}=\rho(x):=\sup _{r>0}\left\{r: \frac{r^{2}}{V(r)} \int_{B(x, r)} W(y) \mathrm{d} y \leq 1\right\} .
$$




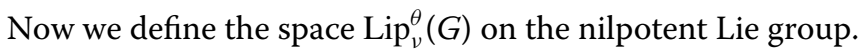

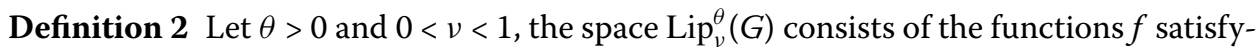
ing

$$
|f(x)-f(y)| \leq C d^{v}(x, y)\left(1+\frac{d(x, y)}{\rho(x)}+\frac{d(x, y)}{\rho(y)}\right)^{\theta}
$$

holds true for all $x, y \in G, x \neq y$. The norm on $\operatorname{Lip}_{v}^{\theta}(G)$ is defined as follows:

$$
\|f\|_{\operatorname{Lip}_{v}^{\theta}(G)} \triangleq \sup _{x, y \in G, x \neq y}\left(\frac{|f(x)-f(y)|}{d^{v}(x, y)\left(1+\frac{d(x, y)}{\rho(x)}+\frac{d(x, y)}{\rho(y)}\right)^{\theta}}\right)<\infty .
$$

It is easy to see that this space is exactly the Lipschitz space when $\theta=0$ if $G$ is a stratified Lie group (cf. [7] and [8]).

We also introduce the following maximal functions.

Definition 3 Let $f \in L_{\mathrm{loc}}^{1}(G)$. For $0<\gamma<D$, the fractional maximal operator is defined by

$$
M_{\gamma} f(x)=\sup _{x \in B} \frac{r^{\gamma}}{|B|} \int_{B}|f(y)| \mathrm{d} y, \quad x \in G,
$$

where the supremum on the right-hand side is taken over all balls $B \subseteq G$ and $r$ is the radius of the ball $B$.

Definition 4 Given $\alpha>0$, the maximal functions for $f \in L_{\mathrm{loc}}^{1}(G)$ and $x \in G$ are defined by

$$
M_{\rho, \alpha} f(x)=\sup _{x \in B \in \mathcal{B}} \frac{1}{|B|} \int_{B}|f(y)| \mathrm{d} y
$$

and

$$
M_{\rho, \alpha}^{\sharp} f(x)=\sup _{x \in B \in \mathcal{B}} \frac{1}{|B|} \int_{B}\left|f(y)-f_{B}\right| \mathrm{d} y,
$$

where $\mathcal{B}_{\rho, \alpha}=\{B(y, r): y \in G$ and $r \leq \alpha \rho(y)\}$.

We are in a position to give the main results in this paper.

Theorem 1 Assume $W \in B_{q_{1}}$ for some $q_{1} \geq \frac{D}{2}$, where $D$ denotes the dimension at infinity of the nilpotent Lie group G. Let

$$
\frac{1}{q_{2}}= \begin{cases}1, & \text { if } q_{1} \geq D, \\ 1-\frac{1}{q_{1}}+\frac{1}{D}, & \text { if } \frac{D}{2} \leq q_{1}<D .\end{cases}
$$

Denote the adjoint operator of $\mathcal{R}$ by $\tilde{\mathcal{R}}=(-\Delta+W)^{-\frac{1}{2}} \nabla$. Then, for any $h \in \operatorname{Lip}_{v}^{\theta}(G), 0<\nu<1$, the commutator $[h, \tilde{\mathcal{R}}]$ is bounded from $L^{q}(G)$ into $L^{p}(G), \frac{1}{p}=\frac{1}{q}-\frac{v}{D}$, if $q_{2}<p<\infty$.

We immediately deduce Corollary 1 by duality. 
Corollary 1 Assume $W \in B_{q_{1}}$ for some $q_{1} \geq \frac{D}{2}$, where $D$ denotes the dimension at infinity of the nilpotent Lie group $G$. Let

$$
\frac{1}{q_{2}}= \begin{cases}1, & \text { if } q_{1} \geq D \\ 1-\frac{1}{q_{1}}+\frac{1}{D}, & \text { if } \frac{D}{2} \leq q_{1}<D .\end{cases}
$$

Then, for any $h \in \operatorname{Lip}_{v}^{\theta}(G), 0<v<1$, the commutator $[h, \mathcal{R}]$ is bounded from $L^{p}(G)$ into $L^{q}(G), \frac{1}{p}=\frac{1}{q}-\frac{v}{D}$, if $1<p<q_{2}^{\prime}$.

Throughout this paper, unless otherwise indicated, $C$ will be used to denote a positive constant that is not necessarily the same case at each occurrence and it depends at most on the constants in (3) and (6). We always denote $\delta=2-\frac{D}{q_{1}}$. By $A \sim B$, we mean that there exist constants $C>0$ and $c>0$ such that $c \leq \frac{A}{B} \leq C$.

\section{Estimates for the kernels of $\mathcal{R}$ and $\tilde{\mathcal{R}}$}

In this section we recall some estimates for the kernels of Riesz transform $\mathcal{R}$ and the dual Riesz transform $\tilde{\mathcal{R}}$, which have been proved in [3].

Lemma $1 W \in B_{q}$ is a doubling measure, that is, there exists a constant $C>0$ such that

$$
\int_{B(x, 2 r)} W(y) \mathrm{d} y \leq C \int_{B(x, r)} W(y) \mathrm{d} y .
$$

Lemma 2 There exists $C>0$ such that, for $0<r<R<\infty$,

$$
\frac{r^{2}}{V(r)} \int_{B(x, r)} W(y) \mathrm{d} y \leq C\left(\frac{R}{r}\right)^{\frac{D}{q}-2} \frac{r^{2}}{V(R)} \int_{B(x, R)} W(y) \mathrm{d} y .
$$

Lemma 3 If $r=\rho(x)$, then

$$
\frac{r^{2}}{V(r)} \int_{B(x, r)} W(y) \mathrm{d} y=1
$$

Moreover,

$$
\frac{r^{2}}{V(r)} \int_{B(x, r)} W(y) \mathrm{d} y \sim 1 \quad \text { if and only if } \quad r \sim \frac{1}{m(x, W)} .
$$

Lemma 4 There exist constants $C, l_{0}>0$ such that

$$
\frac{1}{C}\left(1+\frac{d(x, y)}{\rho(x)}\right)^{-\frac{l_{0}}{l_{0}+1}} \leq \frac{\rho(x)}{\rho(y)} \leq C\left(1+\frac{d(x, y)}{\rho(x)}\right)^{l_{0}} .
$$

In particular, $\rho(x) \sim \rho(y)$ if $d(x, y) \leq C \rho(x)$.

Lemma 5 There exist constants $C>0$ and $l_{1}>0$ such that

$$
\int_{B(x, R)} \frac{d^{2}(x, y) W(y)}{V(d(x, y))} \mathrm{d} y \leq \frac{C R^{2}}{V(R)} \int_{B(x, R)} W(y) \mathrm{d} y \leq C\left(1+\frac{R}{\rho(x)}\right)^{l_{1}} .
$$


Using Lemma 4, we immediately have the following lemma.

Lemma 6 There exist $l_{0}>0, C>0$ such that, for any $x$ and $y$ in $G$,

$$
1+\frac{d(x, y)}{\rho(x)}+\frac{d(x, y)}{\rho(y)} \leq C\left(1+\frac{d(x, y)}{\rho(x)}\right)^{l_{0}+1} .
$$

Let $\Gamma(x, y, \lambda)$ denote the fundamental solution for the operator $-\Delta+W+\lambda$, namely, $[-\Delta+W+\lambda] \Gamma(x, y, \lambda)=\delta\left(y^{-1} x\right)$, where $\delta$ is the Dirac function and $\lambda \in[0, \infty)$. Markedly, $\Gamma(x, y, \lambda)=\Gamma(y, x, \lambda)$.

Lemma 7 Let $N$ be a positive integer.

(i) Suppose $W \in B_{q_{1}}$ for some $q_{1} \geq \frac{D}{2}$. Then there exists a constant $C_{N}>0$ such that, for $x \neq y$,

$$
|\Gamma(x, y, \lambda)| \leq \frac{C_{N}}{\left(1+d(x, y) \lambda^{\frac{1}{2}}\right)^{N}(1+d(x, y) / \rho(x))^{N}} \frac{d(x, y)}{V(d(x, y))} .
$$

(ii) Suppose $W \in B_{q_{1}}$ for some $q_{1} \geq \frac{D}{2}$. Then there exists a constant $C_{N}>0$ such that

$$
\begin{aligned}
\left|\nabla_{x} \Gamma(x, y, \lambda)\right| \leq & \frac{C_{N}}{\left(1+d(x, y) \lambda^{\frac{1}{2}}\right)^{N}(1+d(x, y) / \rho(x))^{N}} \\
& \times\left(\frac{d^{2}(x, y)}{V(d(x, y))} \int_{B(y, d(x, y))} \frac{W(z) d(z, y) \mathrm{d} z}{V(d(z, y))}+\frac{d(x, y)}{V(d(x, y))}\right) .
\end{aligned}
$$

Particularly, if $W \in B_{q_{1}}$ for some $q_{1} \geq D$, then there exists $C_{N}>0$ such that, for $x \neq y$,

$$
\left|\nabla_{x} \Gamma(x, y, \lambda)\right| \leq \frac{C_{N}}{\left(1+d(x, y) \lambda^{\frac{1}{2}}\right)^{N}(1+d(x, y) / \rho(x))^{N}} \frac{d^{2}(x, y)}{V(d(x, y))} .
$$

By the functional calculus, we may write

$$
(-\Delta+W)^{-\frac{1}{2}}=\frac{1}{\pi} \int_{0}^{\infty} \lambda^{-\frac{1}{2}}(-\Delta+W+\lambda)^{-1} \mathrm{~d} \lambda .
$$

Let $f \in C_{0}^{\infty}(G)$. From $(-\Delta+W+\lambda)^{-1} f(x)=\int_{G} \Gamma(x, y, \lambda) f(y) \mathrm{d} y$, it follows that

$$
\mathcal{R} f(x)=\int_{G} \mathcal{K}(x, y) f(y) \mathrm{d} y
$$

where

$$
\mathcal{K}(x, y)=\frac{1}{\pi} \int_{0}^{\infty} \lambda^{-\frac{1}{2}} \nabla_{x} \Gamma(x, y, \lambda) \mathrm{d} \lambda .
$$

Similarly, the adjoint operator of $\mathcal{R}$ is defined to be

$$
\tilde{\mathcal{R}} f(x)=\int_{G} \tilde{\mathcal{K}}(x, y) f(y) \mathrm{d} y,
$$


where

$$
\tilde{\mathcal{K}}(x, y)=\frac{1}{\pi} \int_{0}^{\infty} \lambda^{-\frac{1}{2}} \nabla_{y} \Gamma(y, x, \lambda) \mathrm{d} \lambda .
$$

We recall estimates of the kernels for $\mathcal{R}$ and $\tilde{\mathcal{R}}$ (cf. [3]).

Lemma 8 Suppose $W \in B_{q_{1}}$ for some $\frac{D}{2} \leq q_{1}<D$. For any integer $N>0$, there exists $C_{N}>0$ such that

$$
\begin{aligned}
|\mathcal{K}(x, y)| \leq & \frac{C_{N}}{(1+d(x, y) / \rho(x))^{N}}\left(\frac{d(x, y)}{V(d(x, y))} \int_{B(y, d(x, y))} \frac{W(z) d(z, y) \mathrm{d} z}{V(d(z, y))}\right. \\
& \left.+\frac{1}{V(d(x, y))}\right)
\end{aligned}
$$

and

$$
\begin{aligned}
\left|\mathcal{K}\left(x, y^{\prime}\right)-\mathcal{K}(x, y)\right| \leq & \frac{C_{N} d^{\delta}\left(y, y^{\prime}\right)}{(1+d(x, y) / \rho(x))^{N}} \frac{d^{1-\delta}(x, y)}{V(d(x, y))}\left(\int_{B(y, d(x, y))} \frac{W(z) d(z, y) \mathrm{d} z}{V(d(z, y))}\right. \\
& \left.+\frac{1}{d(x, y)}\right)
\end{aligned}
$$

for some $\delta>0$ and $0<d\left(y, y^{\prime}\right)<\frac{d(x, y)}{16}$. If $W \in B_{q_{1}}$ for some $q_{1} \geq D$, then

$$
|\mathcal{K}(x, y)| \leq \frac{C_{N}}{(1+d(x, y) / \rho(x))^{N}} \frac{1}{V(d(x, y))}
$$

and

$$
\left|\mathcal{K}\left(x, y^{\prime}\right)-\mathcal{K}(x, y)\right| \leq \frac{C_{N}}{(1+d(x, y) / \rho(x))^{N}} \frac{d^{\delta}\left(y, y^{\prime}\right)}{V(d(x, y)) d^{\delta}(x, y)} .
$$

Lemma 9 Suppose $W \in B_{q_{1}}$ for some $\frac{D}{2} \leq q_{1}<D$. For any integer $N>0$, there exists $C_{N}>0$ such that

$$
\begin{aligned}
|\tilde{\mathcal{K}}(x, y)| \leq & \frac{C_{N}}{(1+d(x, y) / \rho(x))^{N}}\left(\frac{d(x, y)}{V(d(x, y))} \int_{B(y, d(x, y))} \frac{W(z) d(z, y) \mathrm{d} z}{V(d(z, y))}\right. \\
& \left.+\frac{1}{V(d(x, y))}\right)
\end{aligned}
$$

and

$$
\begin{aligned}
\left|\tilde{\mathcal{K}}\left(x^{\prime}, y\right)-\tilde{\mathcal{K}}(x, y)\right| \leq & \frac{C_{N} d^{\delta}\left(x, x^{\prime}\right)}{(1+d(x, y) / \rho(x))^{N}} \frac{d^{1-\delta}(x, y)}{V(d(x, y))}\left(\int_{B(y, d(x, y))} \frac{W(z) d(z, y) \mathrm{d} z}{V(d(z, y))}\right. \\
& \left.+\frac{1}{d(x, y)}\right)
\end{aligned}
$$

for some $\delta>0$ and $0<d\left(x, x^{\prime}\right)<\frac{d(x, y)}{16}$. If $W \in B_{q_{1}}$ for some $q_{1} \geq D$, then

$$
|\tilde{\mathcal{K}}(x, y)| \leq \frac{C_{N}}{(1+d(x, y) / \rho(x))^{N}} \frac{1}{V(d(x, y))}
$$


and

$$
\left|\tilde{\mathcal{K}}\left(x^{\prime}, y\right)-\tilde{\mathcal{K}}(x, y)\right| \leq \frac{C_{N}}{(1+d(x, y) / \rho(x))^{N}} \frac{d^{\delta}\left(x, x^{\prime}\right)}{V(d(x, y)) d^{\delta}(x, y)} .
$$

\section{Some technical lemmas and propositions}

Proposition 1 Let $\theta>0$ and $1 \leq s<\infty$. If $h \in \operatorname{Lip}_{v}^{\theta}(G)$, then there exists a positive constant $C$ such that, for all $B=B(x, r)$ with $x \in G$ and $r>0$,

$$
\left(\frac{1}{|B|} \int_{B}\left|h(y)-h_{B}\right|^{s} \mathrm{~d} y\right)^{1 / s} \leq C\|h\|_{\operatorname{Lip}_{\nu}^{\theta}(G)} r^{\nu}\left(1+\frac{r}{\rho(x)}\right)^{\left(l_{0}+1\right) \theta} .
$$

Proof Since $h \in \operatorname{Lip}_{v}^{\theta}(G)$, then

$$
\begin{aligned}
& \left(\frac{1}{|B(x, r)|} \int_{B(x, r)}\left|h(y)-h_{B}\right|^{s} \mathrm{~d} y\right)^{\frac{1}{s}} \\
& \quad \leq\left(\frac{1}{|B(x, r)|} \int_{B(x, r)}|h(y)-h(x)|^{s} \mathrm{~d} y\right)^{\frac{1}{s}}+\left(\frac{1}{|B(x, r)|} \int_{B(x, r)}\left|h(x)-h_{B}\right|^{s} \mathrm{~d} y\right)^{\frac{1}{s}} \\
& \quad \leq 2 C\left(\frac{1}{|B(x, r)|} \int_{B(x, r)}\left|\|h\|_{\operatorname{Lip}_{\nu}^{\theta}(G)} d^{\nu}(x, y)\left(1+\frac{d(x, y)}{\rho(x)}+\frac{d(x, y)}{\rho(y)}\right)^{\theta}\right|^{s} \mathrm{~d} y\right)^{\frac{1}{s}} \\
& \quad \leq C\|h\|_{\operatorname{Lip}_{\nu}^{\theta}(G)} r^{v}\left(1+\frac{r}{\rho(x)}+\frac{r}{\rho(y)}\right)^{\theta} \\
& \quad \leq C\|h\|_{\operatorname{Lip}_{\nu}^{\theta}(G)} r^{v}\left(1+\frac{r}{\rho(x)}\right)^{\left(l_{0}+1\right) \theta},
\end{aligned}
$$

where we have used Lemma 6 in the penultimate inequality.

Similar to the proof of Proposition 1, we immediately get the following.

Lemma 10 Let $h \in \operatorname{Lip}_{v}^{\theta}(G), B=B(x, r)$ and $s \geq 1$. Then there exists a positive constant $C$ such that, for all $k \in N$,

$$
\left(\frac{1}{\left|2^{k} B\right|} \int_{2^{k} B}\left|h(y)-h_{B}\right|^{s} \mathrm{~d} y\right)^{\frac{1}{s}} \leq C\|h\|_{\operatorname{Lip}_{\nu}^{\theta}(G)} 2^{k v} r^{\nu}\left(1+\frac{2^{k} r}{\rho(x)}\right)^{\left(l_{0}+1\right) \theta} .
$$

Proposition 2 Let $W \in B_{q_{1}}$ for $q_{1} \geq \frac{D}{2}$. Let

$$
\frac{1}{q_{2}}= \begin{cases}1, & \text { if } q_{1} \geq D \\ 1-\frac{1}{q_{1}}+\frac{1}{D}, & \text { if } \frac{D}{2} \leq q_{1}<D .\end{cases}
$$

Then there exists $C>0$ such that, for any $f \in C_{0}^{\infty}(G)$,

$$
\|\mathcal{R} f(x)\|_{L^{p}(G)} \leq C\|f\|_{L^{p}(G)},
$$

where $1<p<q_{2}^{\prime}$. 
Proposition 3 Let $W \in B_{q_{1}}$ for $q_{1} \geq \frac{D}{2}$. Let

$$
\frac{1}{q_{2}}= \begin{cases}1, & \text { if } q_{1} \geq D, \\ 1-\frac{1}{q_{1}}+\frac{1}{D}, & \text { if } \frac{D}{2} \leq q_{1}<D .\end{cases}
$$

Then there exists $C>0$ such that, for any $f \in C_{0}^{\infty}(G)$,

$$
\|\tilde{\mathcal{R}} f(x)\|_{L^{p}(G)} \leq C\|f\|_{L^{p}(G)}
$$

where $q_{2}<p<\infty$.

For the proofs of Proposition 2 and Proposition 3, one can refer to [3].

Proposition 4 There exists a sequence of points $\left\{x_{k}\right\}_{k=1}^{\infty}$ in $G$, so that the set of critical balls $Q_{k}=B\left(x_{k}, \rho\left(x_{k}\right)\right), k \geq 1$, satisfies

(i) $\bigcup_{k} Q_{k}=G$;

(ii) There exists $N$ such that, for every $k \in N, \sharp\left\{j: 4 Q_{j} \cap 4 Q_{k} \neq \varnothing\right\} \leq N$.

Proposition 5 For $1<p<\infty$, there exist positive constants $C, \alpha$ and $\beta$ such that if $\left\{Q_{k}\right\}_{k=1}^{\infty}$ is a sequence of balls as in Proposition 4, then

$$
\int_{G}\left|M_{\rho, \alpha} f(x)\right|^{p} \mathrm{~d} x \leq C \int_{G}\left|M_{\rho, \beta}^{\sharp} f(x)\right|^{p} \mathrm{~d} x+C \sum_{k}\left|Q_{k}\right|\left(\frac{1}{\left|Q_{k}\right|} \int_{2 Q_{k}}|f(x)| \mathrm{d} x\right)^{p}
$$

for all $f \in L_{\mathrm{loc}}^{1}(G)$.

The above propositions have been proved in [9] and [10] in the case of a homogeneous space, respectively.

Lemma 11 Let $W \in B_{q_{1}}$ for $q_{1} \geq \frac{D}{2}$, and let

$$
\frac{1}{q_{2}}= \begin{cases}1, & \text { if } q_{1} \geq D, \\ 1-\frac{1}{q_{1}}+\frac{1}{D}, & \text { if } \frac{D}{2} \leq q_{1}<D\end{cases}
$$

and $h \in \operatorname{Lip}_{\nu}^{\theta}(G)$. Then, for $q_{2}<m<\infty$, there exists a positive constant $C$ such that

$$
\frac{1}{|Q|} \int_{Q}|[h, \tilde{\mathcal{R}}] f(x)| \mathrm{d} x \leq C\|h\|_{\operatorname{Lip}_{v}^{\theta}(G)} \inf _{y \in Q}\left\{M_{m v}\left(|f|^{m}\right)(y)\right\}^{\frac{1}{m}}
$$

holds true for all $f \in L_{\mathrm{loc}}^{m}(G)$ and every ball $Q=B\left(x_{0}, \rho\left(x_{0}\right)\right)$, where $M_{m v}$ is a fractional maximal operator.

Proof Throughout the proof of the lemma, we always assume $\frac{D}{2} \leq q_{1}<D$. Let $f \in L^{p}(G)$ and $Q=B\left(x_{0}, \rho\left(x_{0}\right)\right)$. For

$$
[h, \tilde{\mathcal{R}}] f=\left(h-h_{\mathrm{Q}}\right) \tilde{\mathcal{R}} f-\tilde{\mathcal{R}}\left(\left(h-h_{\mathrm{Q}}\right) f\right),
$$


we need to consider the average on $Q$ for each term. By the Hölder inequality with $m>q_{2}$ and Proposition 1,

$$
\begin{aligned}
\frac{1}{|Q|} \int_{Q}\left|\left(h-h_{Q}\right) \tilde{\mathcal{R}} f(x)\right| \mathrm{d} x & \leq\left(\frac{1}{|Q|} \int_{Q}\left|\left(h(x)-h_{Q}\right)\right|^{m^{\prime}} \mathrm{d} x\right)^{1 / m^{\prime}}\left(\frac{1}{|Q|} \int_{Q}|\tilde{\mathcal{R}} f(x)|^{m} \mathrm{~d} x\right)^{1 / m} \\
& \leq C\|h\|_{\operatorname{Lip}_{\nu}^{\theta}(G)}\left(\rho\left(x_{0}\right)\right)^{\nu}\left(\frac{1}{|Q|} \int_{Q}|\tilde{\mathcal{R}} f(x)|^{m} \mathrm{~d} x\right)^{1 / m} .
\end{aligned}
$$

If we write $f=f_{1}+f_{2}$ with $f_{1}=f_{\chi_{2} Q}$, due to Proposition 3 , we get

$$
\begin{aligned}
\left(\rho\left(x_{0}\right)\right)^{v}\left(\frac{1}{|Q|} \int_{Q}\left|\tilde{\mathcal{R}} f_{1}(x)\right|^{m} \mathrm{~d} x\right)^{1 / m} & \leq C\left(\rho\left(x_{0}\right)\right)^{v}\left(\frac{1}{|2 Q|} \int_{2 Q}|f(x)|^{m} \mathrm{~d} x\right)^{1 / m} \\
& \leq C \inf _{y \in Q}\left\{M_{m v}\left(|f|^{m}\right)(y)\right\}^{\frac{1}{m}}
\end{aligned}
$$

for $x \in Q$, and using (17) in Lemma 9, we split $\tilde{\mathcal{R}} f_{2}(x)$ into two parts

$$
\left|\tilde{\mathcal{R}} f_{2}(x)\right|=\left|\int_{d\left(x_{0}, z\right)>2 \rho\left(x_{0}\right)} \tilde{\mathcal{K}}(x, z) f(z) \mathrm{d} z\right| \leq C\left\{I_{1}(x)+I_{2}(x)\right\},
$$

where

$$
I_{1}(x)=\int_{d\left(x_{0}, z\right)>2 \rho\left(x_{0}\right)} \frac{|f(z)|}{V(d(x, z))(1+d(x, z) / \rho(x))^{N}} \mathrm{~d} z
$$

and

$$
I_{2}(x)=\int_{d\left(x_{0}, z\right)>2 \rho\left(x_{0}\right)} \frac{d(x, z)|f(z)|}{V(d(x, z))(1+d(x, z) / \rho(x))^{N}} \int_{B\left(z, \frac{d(x, z)}{4}\right)} \frac{d(u, z) W(u)}{V(d(u, z))} \mathrm{d} u \mathrm{~d} z .
$$

To deal with $I_{1}(x)$, noting that $\rho(x) \sim \rho\left(x_{0}\right)$ and $d(z, x) \sim d\left(z, x_{0}\right)$, we split $d\left(z, x_{0}\right)>2 \rho\left(x_{0}\right)$ into annuli to obtain

$$
\begin{aligned}
\left(\rho\left(x_{0}\right)\right)^{v} I_{1}(x) & =\left(\rho\left(x_{0}\right)\right)^{v} \int_{d\left(x_{0}, z\right)>2 \rho\left(x_{0}\right)} \frac{|f(z)|}{V(d(x, z))(1+d(x, z) / \rho(x))^{N}} \mathrm{~d} z \\
& \leq C \sum_{k \geq 1} \frac{2^{-N k}\left(\rho\left(x_{0}\right)\right)^{v}}{V\left(2^{k} \rho\left(x_{0}\right)\right)} \int_{d\left(x_{0}, z\right)<2^{k} \rho\left(x_{0}\right)}|f(z)| \mathrm{d} z \\
& \leq C \inf _{y \in Q}\left\{M_{m v}\left(|f|^{m}\right)(y)\right\}^{\frac{1}{m}} .
\end{aligned}
$$

Secondly, we consider the term $I_{2}(x)$. We have, for $x \in Q$,

$$
\begin{aligned}
\left(\rho\left(x_{0}\right)\right)^{v} I_{2}(x)= & \left(\rho\left(x_{0}\right)\right)^{v} \int_{d\left(x_{0}, z\right)>2 \rho\left(x_{0}\right)} \frac{d(x, z)|f(z)|}{V(d(x, z))(1+d(x, z) / \rho(x))^{N}} \\
& \times \int_{B\left(z, \frac{d(x, z)}{4}\right)} \frac{d(u, z) W(u)}{V(d(u, z))} \mathrm{d} u \mathrm{~d} z \\
\leq & C\left(\rho\left(x_{0}\right)\right)^{v} \int_{d\left(x_{0}, z\right)>2 \rho\left(x_{0}\right)} \frac{d(x, z)|f(z)|}{V(d(x, z))(1+d(x, z) / \rho(x))^{N}}
\end{aligned}
$$




$$
\begin{aligned}
& \times \int_{B\left(z, 4 d\left(x_{0}, z\right)\right)} \frac{d(u, z) W(u)}{V(d(u, z))} \mathrm{d} u \mathrm{~d} z \\
\leq & C\left(\rho\left(x_{0}\right)\right)^{v} \sum_{k \geq 1} \frac{2^{-N k} 2^{k} \rho\left(x_{0}\right)}{V\left(2^{k} \rho\left(x_{0}\right)\right)} \int_{d\left(x_{0}, z\right)<2^{k+1} \rho\left(x_{0}\right)}|f(z)| \\
& \times \int_{B\left(z, 2^{k+3} d\left(x_{0}, z\right)\right)} \frac{d(u, z) W(u)}{V(d(u, z))} \mathrm{d} u \mathrm{~d} z \\
\leq & C\left(\rho\left(x_{0}\right)\right)^{v} \sum_{k \geq 1} \frac{2^{-N k} 2^{k} \rho\left(x_{0}\right)}{V\left(2^{k} \rho\left(x_{0}\right)\right)} \\
& \times \int_{d\left(x_{0}, z\right)<2^{k+1} \rho\left(x_{0}\right)}|f(z)| \mathcal{I}_{1}\left(W_{\chi_{B\left(x_{0}, 2^{k} \rho\left(x_{0}\right)\right)}}\right)(z) \mathrm{d} z .
\end{aligned}
$$

Let $q_{2}<m<D$. Using the Hölder inequality and the boundedness of the fractional inte$\operatorname{gral} \mathcal{I}_{1}: L^{m^{\prime}} \mapsto L^{q_{1}}$ with

$$
\frac{1}{q_{1}}=\frac{1}{m^{\prime}}-\frac{1}{\beta}
$$

where

$$
\beta= \begin{cases}d, & \text { if } 2^{k} \rho\left(x_{0}\right)<1, \\ D, & \text { if } 2^{k} \rho\left(x_{0}\right) \geq 1\end{cases}
$$

(cf. Theorem 1.6 in [11]), we obtain

$$
\begin{aligned}
& \int_{d\left(x_{0}, z\right)<2^{k+1} \rho\left(x_{0}\right)}|f(z)| \mathcal{I}_{1}\left(W \chi_{B\left(x_{0}, 2^{k} \rho\left(x_{0}\right)\right)}\right)(z) \mathrm{d} z \\
& \quad \leq\left\|f \chi_{B\left(x_{0}, 2^{k} \rho\left(x_{0}\right)\right)}\right\|_{m} \| \mathcal{I}_{1}\left(W \chi_{\left.B\left(x_{0}, 2^{k} \rho\left(x_{0}\right)\right)\right)} \|_{m^{\prime}}\right. \\
& \quad \leq\left\|f \chi_{B\left(x_{0}, 2^{k} \rho\left(x_{0}\right)\right)}\right\|_{m}\left\|W \chi_{B\left(x_{0}, 2^{k} \rho\left(x_{0}\right)\right)}\right\|_{q_{1}} .
\end{aligned}
$$

Since $W \in B_{q_{1}}$, we obtain

$$
\begin{aligned}
\left\|W \chi_{B\left(x_{0}, 2^{k} \rho\left(x_{0}\right)\right)}\right\|_{q_{1}} & \leq C\left(V\left(2^{k} \rho\left(x_{0}\right)\right)\right)^{-\frac{1}{q_{1}^{\prime}}} \int_{B\left(x_{0}, 2^{k} \rho\left(x_{0}\right)\right)} W \\
& \leq C\left(2^{k} \rho\left(x_{0}\right)\right)^{-2}\left(V\left(2^{k} \rho\left(x_{0}\right)\right)\right)^{1-\frac{1}{q_{1}^{\prime}}} \frac{\left(2^{k} \rho\left(x_{0}\right)\right)^{2}}{V\left(2^{k} \rho\left(x_{0}\right)\right)} \int_{B\left(x_{0}, 2^{k} \rho\left(x_{0}\right)\right)} W \\
& \leq C\left(2^{k}\right)^{l_{1}-2}\left(V\left(2^{k} \rho\left(x_{0}\right)\right)\right)^{1-\frac{1}{q_{1}^{\prime}}}\left(\rho\left(x_{0}\right)\right)^{-2},
\end{aligned}
$$

where in the last two inequalities we have used doubling measure and the definition of $\rho$, respectively. Therefore,

$$
I_{2}(x) \leq V\left(\rho\left(x_{0}\right)\right)^{-1 / q_{1}^{\prime}-1} \sum_{k \geq 1} 2^{k\left(-N+l_{1}-1-d / q_{1}^{\prime}\right)}\left\|f \chi_{B\left(x_{0}, 2^{k} \rho\left(x_{0}\right)\right)}\right\|_{m} .
$$

Finally, observing that

$$
\left\|f \chi_{B\left(x_{0}, 2^{k} \rho\left(x_{0}\right)\right)}\right\|_{m} \leq\left(V\left(2^{k} \rho\left(x_{0}\right)\right)\right)^{\frac{1}{m}} \inf _{y \in Q} M_{m} f(y)
$$


and using that $\frac{1}{q_{1}^{\prime}}-\frac{1}{m}=\frac{1}{D}$ or $\frac{1}{d}$, we have

$$
\left(\rho\left(x_{0}\right)\right)^{v} I_{2}(x) \leq C \inf _{y \in Q}\left\{M_{m v}\left(|f|^{m}\right)(y)\right\}^{\frac{1}{m}}
$$

by choosing $N$ large enough.

So far, we have solved the term $\left(h-h_{Q}\right) \mathcal{R} f$, now we want to control $\int \mathcal{R}\left[\left(h-h_{Q}\right) f\right] \mathrm{d} x$ by the term $C \inf _{y \in Q}\left\{M_{m v}\left(|f|^{m}\right)(y)\right\}^{\frac{1}{m}}$. We still split $f=f_{1}+f_{2}$. Choose $q_{2}<\tilde{m}<m$ and set $t=\frac{\tilde{m} m}{m-\tilde{m}}$. Using the boundedness of $\tilde{\mathcal{R}}$ on $L^{\tilde{m}}(G)$ and the Hölder inequality, we get

$$
\begin{aligned}
& \frac{1}{|Q|} \int_{Q}\left|\tilde{\mathcal{R}}\left(h-h_{Q}\right) f_{1}(x)\right| \mathrm{d} x \\
& \quad \leq\left(\frac{1}{|Q|} \int_{Q}\left|\tilde{\mathcal{R}}\left(h-h_{Q}\right) f_{1}(x)\right|^{\tilde{m}} \mathrm{~d} x\right)^{1 / \tilde{m}} \\
& \quad \leq C\left(\frac{1}{|Q|} \int_{Q}\left|\left(h-h_{Q}\right) f(x)\right|^{\tilde{m}} \mathrm{~d} x\right)^{1 / \tilde{m}} \\
& \quad \leq C\left(\frac{1}{|Q|} \int_{2 Q}|f(x)|^{m} \mathrm{~d} x\right)^{1 / m}\left(\frac{1}{|Q|} \int_{2 Q}\left|h(x)-h_{Q}\right|^{t} \mathrm{~d} x\right)^{1 / t} \\
& \quad \leq C\left(\frac{\left(\rho\left(x_{0}\right)\right)^{m v}}{|Q|} \int_{2 Q}|f(x)|^{m} \mathrm{~d} x\right)^{1 / m}\|h\|_{\operatorname{Lip}_{v}^{\theta}(G)}\left(1+\frac{\rho\left(x_{0}\right)}{\rho\left(x_{0}\right)}\right)^{\left(l_{0}+1\right) \theta} \\
& \quad \leq C\|h\|_{\operatorname{Lip}_{v}^{\theta}(G)} \inf _{y \in Q}\left\{M_{m v}\left(|f|^{m}\right)(y)\right\}^{\frac{1}{m}},
\end{aligned}
$$

where we have applied Proposition 1 to the last but one inequality. Similarly, for $x \in Q$ and using (17) in Lemma 9, we have

$$
\left|\tilde{R}\left(h-h_{Q}\right) f_{2}(x)\right|=\left|\int_{d\left(x_{0}, z\right)>2 \rho\left(x_{0}\right)} \tilde{\mathcal{K}}(x, z)\left(h(z)-h_{Q}\right) f(z) \mathrm{d} z\right| \leq C\left\{\tilde{I}_{1}(x)+\tilde{I}_{2}(x)\right\},
$$

where

$$
\tilde{I}_{1}(x)=\int_{d\left(x_{0}, z\right)>2 \rho\left(x_{0}\right)} \frac{\left|\left(h-h_{Q}\right) f(z)\right|}{V(d(x, z))(1+d(x, z) / \rho(x))^{N}} \mathrm{~d} z
$$

and

$$
\tilde{I}_{2}(x)=\int_{d\left(x_{0}, z\right)>2 \rho\left(x_{0}\right)} \frac{\left|\left(h-h_{Q}\right) f(z)\right|}{V(d(x, z))(1+d(x, z) / \rho(x))^{N}} \int_{B\left(z, \frac{d(x, z)}{4}\right)} \frac{d(u, z) W(u)}{V(d(u, z))} \mathrm{d} u \mathrm{~d} z .
$$

We start by observing that for $1 \leq \tilde{m}<m, t=\frac{\tilde{m} m}{m-\tilde{m}}$, and by Lemma 6 ,

$$
\begin{aligned}
& \left\|\left[\left(h-h_{Q}\right) f\right] \chi_{B\left(x_{0}, 2^{k} \rho\left(x_{0}\right)\right)}\right\|_{\tilde{m}} \\
& \quad \leq\left\|f_{\chi_{B\left(x_{0}, 2^{k} \rho\left(x_{0}\right)\right)}}\right\| m\left\|\left(h-h_{Q}\right) \chi_{B\left(x_{0}, 2^{k} \rho\left(x_{0}\right)\right)}\right\|_{t} \\
& \quad \leq C\left(\int_{B\left(x_{0}, 2^{k} \rho\left(x_{0}\right)\right)}|f(y)|^{m} \mathrm{~d} y\right)^{1 / m}\|h\|_{\operatorname{Lip}_{\nu}^{\theta}(G)}\left(2^{k} \rho\left(x_{0}\right)\right)^{v}\left(1+2^{k}\right)^{\left(l_{0}+1\right) \theta} V^{1 / t}\left(2^{k} \rho\left(x_{0}\right)\right) \\
& \quad \leq C 2^{k\left(l_{0}+1\right) \theta} V^{1 / \tilde{m}}\left(2^{k} \rho\left(x_{0}\right)\right)\|h\|_{\operatorname{Lip}_{v}^{\theta}(G)} \inf _{y \in Q}\left\{M_{m v}\left(|f|^{m}\right)(y)\right\}^{\frac{1}{m}} .
\end{aligned}
$$


For $\tilde{I}_{1}(x)$, using (24) with $\tilde{m}=1$, we have

$$
\begin{aligned}
\tilde{I}_{1}(x) & \leq C \sum_{k \geq 1} \frac{2^{-N k}}{V\left(2^{k} \rho\left(x_{0}\right)\right)} \int_{d\left(x_{0}, z\right)>2^{k} \rho\left(x_{0}\right)}\left|h(z)-h_{Q}\right||f(z)| \mathrm{d} z \\
& \leq C\|h\|_{\operatorname{Lip}_{\nu}^{\theta}(G)} \inf _{y \in Q}\left\{M_{m v}\left(|f|^{m}\right)(y)\right\}^{\frac{1}{m}} \sum_{k \geq 1} 2^{k\left(-N+\left(l_{0}+1\right) \theta\right)} \\
& \leq C\|h\|_{\operatorname{Lip}_{\nu}^{\theta}(G)} \inf _{y \in Q}\left\{M_{m v}\left(|f|^{m}\right)(y)\right\}^{\frac{1}{m}}
\end{aligned}
$$

if we choose $N$ sufficiently large.

To deal with $\tilde{I}_{2}(x)$, we discuss as in the estimate for $I_{2}(x)$ with $\left(h-h_{Q}\right) f$ instead of $f$ and $\tilde{m}$ and $\tilde{q}_{1}$ instead of $m$ and $q_{1}$, but we cannot avoid to discuss the different cases where $2^{k} \rho\left(x_{0}\right) \geq 1$ and $2^{k} \rho\left(x_{0}\right)<1$. Let

$$
\frac{1}{\tilde{m}}= \begin{cases}\frac{1}{\tilde{q_{1}}}-\frac{1}{d}, & \text { if } 2^{k} \rho\left(x_{0}\right)<1, \\ \frac{1}{\tilde{q_{1}}}-\frac{1}{D}, & \text { if } 2^{k} \rho\left(x_{0}\right) \geq 1\end{cases}
$$

Using (24), we similarly have

$$
\tilde{I}_{2}(x) \leq C\|h\|_{\operatorname{Lip}_{v}^{\theta}(G)} \inf _{y \in Q}\left\{M_{m v}\left(|f|^{m}\right)(y)\right\}^{\frac{1}{m}}
$$

where we choose $N$ large enough to ensure the above series converges.

Lemma 12 Let $\tilde{\mathcal{R}}=(-\Delta+W)^{-\frac{1}{2}} \nabla$ be the adjoint operator of the Riesz transform $\mathcal{R}$. Then there exists $C>0$ such that, for any $f \in L_{\mathrm{loc}}^{m}(G)$ and $h \in \operatorname{Lip}_{v}^{\theta}(G)$,

$$
M_{\rho, \alpha}^{\sharp}([h, \tilde{R}] f)(x) \leq C\|h\|_{\operatorname{Lip}_{v}^{\theta}(G)}\left(\left\{M_{m v}\left(|f|^{m}\right)(y)\right\}^{\frac{1}{m}}+\left\{M_{m v}\left(|\tilde{\mathcal{R}} f|^{m}\right)(x)\right\}^{\frac{1}{m}}\right) .
$$

Proof Let $f \in L_{\mathrm{loc}}^{m}(G), x \in G$ and a ball $B=B\left(x_{0}, r\right)$ with $x \in B$ and $r<\epsilon \rho\left(x_{0}\right), \epsilon>0$, we need to control $J=\frac{1}{|B|} \int_{B}|[h, \tilde{\mathcal{R}}] f(y)-c| \mathrm{d} y$ by the right-hand side of (25) for some constant $c$, which will be designated later. Let $f=f_{1}+f_{2}$, where $f_{1}=f_{\chi_{2 B}}$ and $f_{2}=f-f_{1}$. Then

$$
\begin{aligned}
{[h, \tilde{\mathcal{R}}] f } & =\left[h-h_{2 B}, \tilde{\mathcal{R}}\right] f \\
& =\left(h-h_{2 B}\right) \tilde{\mathcal{R}} f-\tilde{\mathcal{R}}\left(h-h_{2 B}\right) f_{1}-\tilde{\mathcal{R}}\left(h-h_{2 B}\right) f_{2} \\
& \doteq A_{1} f+A_{2} f+A_{3} f .
\end{aligned}
$$

Take $c=\int_{d\left(x_{0}, z\right) \geq 2 r} \tilde{\mathcal{K}}\left(x_{0}, z\right)\left(h(z)-h_{2 B}\right) f(z) \mathrm{d} z$. Then we have

$$
\begin{aligned}
J & \leq \frac{1}{|B|} \int_{B}\left|A_{1} f(y)\right| \mathrm{d} y+\frac{1}{|B|} \int_{B}\left|A_{2} f(y)\right| \mathrm{d} y+\frac{1}{|B|} \int_{B}\left|A_{3} f(y)-c\right| \mathrm{d} y \\
& \doteq J_{1}+J_{2}+J_{3} .
\end{aligned}
$$

At first, we consider $J_{1}$. Note that $d\left(x, x_{0}\right) \leq r<\epsilon \rho\left(x_{0}\right)$ implies $\rho(x) \sim \rho\left(x_{0}\right)$. By the Hölder 
Ni and Li Journal of Inequalities and Applications <wide>(2017) 2017:312

Page 13 of 17

inequality, Proposition 1 and Proposition 3, we have

$$
\begin{aligned}
J_{1} & =\frac{1}{|B|} \int_{B}\left|A_{1} f(y)\right| \mathrm{d} y \\
& =\frac{1}{|B|} \int_{B}\left|\left(h(y)-h_{2 B}\right) \tilde{\mathcal{R}} f(y)\right| \mathrm{d} y \\
& \leq \frac{C}{|B|}\left[\int_{B}\left|h(y)-h_{2 B}\right|^{\frac{m}{m-1}} \mathrm{~d} y\right]^{\frac{m-1}{m}}\left[\int_{B}|\tilde{\mathcal{R}} f(y)|^{m} \mathrm{~d} y\right]^{\frac{1}{m}} \\
& \leq \frac{C}{|B|}\left[\int_{2 B}\left|h(y)-h_{2 B}\right|^{\frac{m}{m-1}} \mathrm{~d} y\right]^{\frac{m-1}{m}}\left[\int_{B}|\tilde{\mathcal{R}} f(y)|^{m} \mathrm{~d} y\right]^{\frac{1}{m}} \\
& \leq \frac{C}{|B|}\|h\|_{\operatorname{Lip}_{v}^{\theta}(G)}\left(1+\frac{2 r}{\rho\left(x_{0}\right)}\right)^{\left(l_{0}+1\right) \theta} r^{v}|B|^{\frac{m-1}{m}}\left[\int_{B}|\tilde{\mathcal{R}} f(y)|^{m} \mathrm{~d} y\right]^{\frac{1}{m}} \\
& \leq \frac{C}{|B|}\|h\|_{\operatorname{Lip}_{v}^{\theta}(G)}\left(1+\frac{2 \epsilon \rho\left(x_{0}\right)}{\rho\left(x_{0}\right)}\right)^{\left(l_{0}+1\right) \theta} r^{\nu}|B|^{\frac{m-1}{m}}\left[\int_{B}|\tilde{\mathcal{R}} f(y)|^{m} \mathrm{~d} y\right]^{\frac{1}{m}} \\
& \leq C^{\prime}\|h\|_{\operatorname{Lip}_{\nu}^{\theta}(G)}\left(\frac{r^{m v}}{|B|} \int_{B}|\tilde{\mathcal{R}} f(y)|^{m} \mathrm{~d} y\right)^{\frac{1}{m}} \\
& \leq C^{\prime}\|h\|_{\operatorname{Lip}_{\nu}^{\theta}(G)}\left\{M_{m v}\left(|\tilde{\mathcal{R}} f|^{m}\right)(x)\right\}^{\frac{1}{m}}
\end{aligned}
$$

for $m>q_{2}$.

For $J_{2}$, by the Hölder inequality and Proposition 3,

$$
\begin{aligned}
& J_{2} \leq \frac{1}{|B|} \int_{B}\left|A_{2} f(y)\right| \mathrm{d} y \\
& \leq\left(\frac{1}{|B|} \int_{B}\left|A_{2} f(y)\right|^{\tilde{m}} \mathrm{~d} y\right)^{\frac{1}{\tilde{m}}} \\
& \leq C\left(\frac{1}{|B|}\right)^{\frac{1}{\tilde{m}}}\left(\int_{2 B}\left|\left(h(y)-h_{2 B}\right) f(y)\right|^{\tilde{m}} \mathrm{~d} y\right)^{\frac{1}{\tilde{m}}} \\
& \leq \frac{C}{|B|^{\frac{1}{\tilde{m}}}}\left(\int_{2 B}\left|h(y)-h_{2 B}\right|^{\frac{m \tilde{m}}{m-\tilde{m}}} \mathrm{~d} y\right)^{\frac{1}{\tilde{m}}-\frac{1}{m}}\left(\int_{2 B}|f(y)|^{m} \mathrm{~d} y\right)^{\frac{1}{m}} \\
& \leq C\|h\|_{\operatorname{Lip}_{\nu}^{\theta}(G)} r^{\nu}\left(1+\frac{2 r}{\rho\left(x_{0}\right)}\right)^{\left(l_{0}+1\right) \theta}\left(\frac{1}{|B|}\right)^{\frac{1}{m}}\left(\int_{2 B}|f(y)|^{m} \mathrm{~d} y\right)^{\frac{1}{m}} \\
& \leq C\|h\|_{\operatorname{Lip}_{v}^{\theta}(G)} r^{v}\left(1+\frac{2 \epsilon \rho\left(x_{0}\right)}{\rho\left(x_{0}\right)}\right)^{\left(l_{0}+1\right) \theta}\left(\frac{1}{|B|}\right)^{\frac{1}{m}}\left(\int_{2 B}|f(y)|^{m} \mathrm{~d} y\right)^{\frac{1}{m}} \\
& \leq C^{\prime}\|h\|_{\operatorname{Lip}_{v}^{\theta}(G)}\left(\frac{r^{m v}}{|B|} \int_{2 B}|f(y)|^{m} \mathrm{~d} y\right)^{\frac{1}{m}} \\
& \leq C^{\prime}\|h\|_{\operatorname{Lip}_{v}^{\theta}(G)}\left(\frac{(2 r)^{m v}}{|2 B|} \int_{2 B}|f(y)|^{m} \mathrm{~d} y\right)^{\frac{1}{m}} \\
& \leq C^{\prime}\|h\|_{\operatorname{Lip}_{\nu}^{\theta}(G)}\left\{M_{m v}\left(|f|^{m}\right)(y)\right\}^{\frac{1}{m}},
\end{aligned}
$$

where $q_{2}<\tilde{m}<m$. 
Finally, we consider $J_{3}$.

Case of $q_{1} \geq D$ : By Lemmas 9 and 6, we have

$$
\begin{aligned}
& \left(\int_{2^{k} r \leq d\left(x_{0}, z\right)<2^{k+1} r}\left|\tilde{\mathcal{K}}(y, z)-\tilde{\mathcal{K}}\left(x_{0}, z\right)\right|^{m^{\prime}} \mathrm{d} z\right)^{\frac{1}{m^{\prime}}}\left(\int_{2^{k} r \leq d\left(x_{0}, z\right)<2^{k+1} r}\left|h(z)-h_{2 B}\right|^{s^{\prime}} \mathrm{d} z\right)^{\frac{1}{s^{\prime}}} \\
& \leq \frac{C_{N} r^{\delta}}{\left(1+2^{k} r / \rho\left(x_{0}\right)\right)^{N}}\left(\int_{2^{k} r \leq d\left(x_{0}, z\right)<2^{k+1} r} \frac{1}{d^{m^{\prime} \delta}\left(x_{0}, z\right) V^{m^{\prime}}\left(d\left(x_{0}, z\right)\right)} \mathrm{d} z\right)^{\frac{1}{m^{\prime}}} \\
& \times\left(\int_{d\left(x_{0}, z\right)<2^{k+1} r}\left|h(z)-h_{2 B}\right|^{s^{\prime}} \mathrm{d} z\right)^{\frac{1}{s^{\prime}}} \\
& \leq \frac{C_{N}}{\left(1+2^{k} r / \rho\left(x_{0}\right)\right)^{N}} \frac{r^{\delta}}{\left(2^{k} r\right)^{\delta} V^{\left(m^{\prime}-1\right) / m\left(2^{k} r\right)}}\|h\|_{\operatorname{Lip}_{\nu}^{\theta}(G)} 2^{k v} r^{v}\left(1+\frac{2^{k} r}{\rho\left(x_{0}\right)}\right)^{\left(l_{0}+1\right) \theta} V^{\frac{1}{s}}\left(2^{k} r\right) \\
& \leq\|h\|_{\operatorname{Lip}_{v}^{\theta}(G)} 2^{k v} r^{v} \frac{C_{N}}{\left(1+2^{k} r / \rho\left(x_{0}\right)\right)^{N-\left(l_{0}+1\right) \theta}} \frac{r^{\delta}}{\left(2^{k} r\right)^{\delta} V^{1 / m}\left(2^{k} r\right)},
\end{aligned}
$$

where $\frac{1}{m}+\frac{1}{m^{\prime}}+\frac{1}{s}=1$ and $\frac{1}{s^{\prime}}+\frac{1}{s}=1$. Therefore, via the Hölder inequality,

$$
\begin{aligned}
& J_{3}=\frac{1}{|B|} \int_{B}\left|A_{3} f(y)-c\right| \mathrm{d} y \\
& \leq \frac{1}{|B|} \int_{B}\left|\int_{d\left(x_{0}, z\right)>2 r}\left(\tilde{\mathcal{K}}(y, z)-\tilde{\mathcal{K}}\left(x_{0}, z\right)\right)\left(h(z)-h_{2 Q}\right) f(z) \mathrm{d} z\right| \mathrm{d} y \\
& \leq \frac{1}{|B|} \int_{B} \sum_{k=1}^{\infty} \int_{2^{k} r \leq d\left(x_{0}, z\right)<2^{k+1} r}\left|\left(\tilde{\mathcal{K}}(y, z)-\tilde{\mathcal{K}}\left(x_{0}, z\right)\right)\left(h(z)-h_{2 Q}\right) f(z)\right| \mathrm{d} z \mathrm{~d} y \\
& \leq \frac{\|h\|_{\operatorname{Lip}_{v}^{\theta}(G)}}{|B|} \int_{B} \sum_{k=1}^{\infty} 2^{k v} r^{\nu} \frac{C}{\left(1+2^{k} r / \rho\left(x_{0}\right)\right)^{N-\left(l_{0}+1\right) \theta}} \frac{r^{\delta}}{\left(2^{k} r\right)^{\delta} V^{\frac{1}{m}-\frac{1}{s}}\left(2^{k} r\right)} \\
& \times\left(\int_{d\left(x_{0}, z\right)<2^{k+1} r}|f(z)|^{m} \mathrm{~d} z\right)^{\frac{1}{m}} \\
& \leq C\|h\|_{\operatorname{Lip}_{\nu}^{\theta}(G)} \sum_{k=1}^{\infty} \frac{C_{N}}{\left(1+2^{k} r / \rho\left(x_{0}\right)\right)^{N-\left(l_{0}+1\right) \theta}} \frac{r^{\delta}}{\left(2^{k} r\right)^{\delta} V^{1 / m}\left(2^{k} r\right)} \frac{\left(2^{k} r\right)^{\nu}}{\left(2^{k+1} r\right)^{\nu}} V^{\frac{1}{m}}\left(2^{k+1} r\right) \\
& \times\left(\frac{\left(2^{k+1} r\right)^{m v}}{V\left(2^{k+1} r\right)} \int_{d\left(x_{0}, z\right)<2^{k+1} r}|f(z)|^{m} \mathrm{~d} z\right)^{\frac{1}{m}} \\
& \leq C\|h\|_{\operatorname{Lip}_{\nu}^{\theta}(G)} \sum_{k=1}^{\infty} 2^{-k \delta} \frac{C_{N}}{\left(1+2^{k} r / \rho\left(x_{0}\right)\right)^{N-\left(l_{0}+1\right) \theta}}\left\{M_{m v}\left(|f|^{m}\right)(y)\right\}^{\frac{1}{m}} \\
& \leq C\|h\|_{\operatorname{Lip}_{\nu}^{\theta}(G)}\left\{M_{m v}\left(|f|^{m}\right)(y)\right\}^{\frac{1}{m}}
\end{aligned}
$$

if we choose $N$ sufficiently large.

Case of $\frac{D}{2} \leq q_{1}<D$ : By Lemmas 9 and 6 , we have

$$
\begin{aligned}
& \left(\int_{2^{k} r \leq d\left(x_{0}, z\right)<2^{k+1} r}\left|\tilde{\mathcal{K}}(y, z)-\tilde{\mathcal{K}}\left(x_{0}, z\right)\right|^{p} \mathrm{~d} z\right)^{\frac{1}{p}}\left(\int_{2^{k} r \leq d\left(x_{0}, z\right)<2^{k+1} r}\left|h(z)-h_{2 B}\right|^{t} \mathrm{~d} z\right)^{\frac{1}{t}} \\
& \quad \leq \frac{C_{N} r^{\delta}\left(2^{k} r\right)^{1-\delta}}{V\left(2^{k} r\right)\left(1+2^{k} r / \rho\left(x_{0}\right)\right)^{N}}\left(\int_{2^{k} r \leq d\left(x_{0}, z\right)<2^{k+1_{r}}}\left|\int_{B\left(z, 2^{k+3} r\right)} \frac{d(z, y) W(y)}{V(z, y)} \mathrm{d} y\right|^{p} \mathrm{~d} z\right)^{\frac{1}{p}}
\end{aligned}
$$




$$
\begin{aligned}
& \times\left(\int_{2^{k} r \leq d\left(x_{0}, z\right)<2^{k+1} r}\left|h(z)-h_{2 B}\right|^{t} \mathrm{~d} z\right)^{\frac{1}{t}} \\
& +\frac{C_{N}\|h\|_{\operatorname{Lip}_{v}^{\theta}(G)} 2^{l v} r^{v}}{\left(1+2^{k} r / \rho\left(x_{0}\right)\right)^{N-\left(l_{0}+1\right) \theta}} \frac{r^{\delta}}{\left(2^{k} r\right)^{\delta} V^{1 / m}\left(2^{k} r\right)} \\
\leq & \frac{\left.C_{N}\|h\|_{\operatorname{Lip}_{v}^{\theta}(G)}\right)^{l v} r^{v}}{\left(1+2^{k} r / \rho\left(x_{0}\right)\right)^{N-\left(l_{0}+1\right) \theta}} \\
& \times\left[\frac{r^{\delta}\left(2^{k} r\right)^{1-\delta}}{V^{\frac{t-1}{t}}\left(2^{k} r\right)}\left(\int_{B\left(x_{0}, 2^{k+3} r\right)} W^{q_{1}}(z) \mathrm{d} z\right)^{\frac{1}{q_{1}}}+\frac{r^{\delta}}{\left(2^{k} r\right)^{\delta} V^{1 / m}\left(2^{k} r\right)}\right] \\
\leq & \frac{C_{N}\|h\|_{\operatorname{Lip}_{v}^{\theta}(G)} 2^{l v} r^{v}}{\left(1+2^{k} r \rho \rho\left(x_{0}\right)\right)^{N-\left(l_{0}+1\right) \theta}} \\
& \times\left[\frac{r^{\delta} V^{\frac{1}{q_{1}}}\left(2^{k} r\right)\left(2^{k} r\right)^{-1-\delta}}{V^{t-1} t\left(2^{k} r\right)}\left(\frac{\left(2^{k+3} r\right)^{2}}{V\left(2^{k+3} r\right)} \int_{B\left(x_{0}, 2^{k+3} r\right)} W(z) \mathrm{d} z\right)+\frac{r^{\delta}}{\left(2^{k} r\right)^{\delta} V^{1 / m}\left(2^{k} r\right)}\right] \\
\leq & \frac{C_{N}\|h\|_{\operatorname{Lip}_{v}^{\theta}(G)} 2^{l v} r^{v}}{\left(1+2^{k} r \rho \rho\left(x_{0}\right)\right)^{N-l_{1}-\left(l_{0}+1\right) \theta}}\left(\frac{r^{\delta} V^{\frac{1}{q_{1}}}\left(2^{k} r\right)\left(2^{k} r\right)^{-1-\delta}}{V^{\frac{t-1}{t}}\left(2^{k} r\right)}+\frac{r^{\delta}}{\left(2^{k} r\right)^{\delta} V^{1 / m}\left(2^{k} r\right)}\right) \\
\leq & \frac{C\|h\|_{\operatorname{Lip}_{v}^{\theta}(G)} 2^{l v} r^{v}}{\left(1+2^{k} r \rho \rho\left(x_{0}\right)\right)^{N-l_{1}-\left(l_{0}+1\right) \theta}} \frac{r^{\delta}}{\left(2^{k} r\right)^{\delta} V^{1 / m}\left(2^{k} r\right)},
\end{aligned}
$$

where $\frac{1}{m}+\frac{1}{p}+\frac{1}{t}=1$ and $\frac{1}{q_{1}}=\frac{1}{p}+\frac{1}{D}$. Therefore, for $m>q_{2}$,

$$
\begin{aligned}
J_{3}= & \frac{1}{|B|} \int_{B}\left|A_{3} f(y)-c\right| \mathrm{d} y \\
\leq & \frac{1}{|B|} \int_{B}\left|\int_{d\left(x_{0}, z\right)>2 r}\left(\tilde{\mathcal{K}}(y, z)-\tilde{\mathcal{K}}\left(x_{0}, z\right)\right)\left(h(z)-h_{2 Q}\right) f(z) \mathrm{d} z\right| \mathrm{d} y \\
\leq & \frac{1}{|B|} \int_{B} \sum_{k=1}^{\infty} \int_{2^{k} r \leq d\left(x_{0}, z\right)<2^{k+1} r}\left|\left(\tilde{\mathcal{K}}(y, z)-\tilde{\mathcal{K}}\left(x_{0}, z\right)\right)\left(h(z)-h_{2 Q}\right) f(z)\right| \mathrm{d} z \mathrm{~d} y \\
\leq & \frac{\|h\|_{\operatorname{Lip}_{v}^{\theta}(G)}}{|B|} \int_{B} \sum_{k=1}^{\infty} 2^{k v} r^{\nu} \frac{C}{\left(1+2^{k} r / \rho\left(x_{0}\right)\right)^{N-l_{1}-\left(l_{0}+1\right) \theta}} \frac{r^{\delta}}{\left(2^{k} r\right)^{\delta} V^{\frac{1}{m}-\frac{1}{s}\left(2^{k} r\right)}} \\
& \times\left(\int_{d\left(x_{0}, z\right)<2^{k+1} r}|f(z)|^{m} \mathrm{~d} z\right)^{\frac{1}{m}} \\
\leq & C\|h\|_{\operatorname{Lip}_{\nu}^{\theta}(G)} \sum_{k=1}^{\infty} \frac{C_{N}}{\left(1+2^{k} r / \rho\left(x_{0}\right)\right)^{N-l_{1}-\left(l_{0}+1\right) \theta}} \frac{r^{\delta}}{\left(2^{k} r\right)^{\delta} V^{1 / m}\left(2^{k} r\right)} \frac{\left(2^{k} r\right)^{\nu}}{\left(2^{k+1} r\right)^{\nu}} V^{\frac{1}{m}}\left(2^{k+1} r\right) \\
& \times\left(\left.\frac{\left(2^{k+1} r\right)^{m v}}{V\left(2^{k+1} r\right)} \int_{d\left(x_{0}, z\right)<2^{k+1} r} f(z)\right|^{m} \mathrm{~d} z\right)^{\frac{1}{m}} \\
\leq & C\|h\|_{\operatorname{Lip}_{\nu}^{\theta}(G)} \sum_{k=1}^{\infty} 2^{-k \delta} \frac{C_{N}}{\left(1+2^{k} r / \rho\left(x_{0}\right)\right)^{N-l_{1}-\left(l_{0}+1\right) \theta}}\left\{M_{m v}\left(|f|^{m}\right)(y)\right\}^{\frac{1}{m}} \\
\leq & C\|h\|_{\operatorname{Lip}_{\nu}^{\theta}(G)}\left\{M_{m v}\left(|f|^{m}\right)(y)\right\}^{\frac{1}{m}},
\end{aligned}
$$

if we choose $N$ sufficiently large. 


\section{Proof of the main result}

Proof of Theorem 1 Suppose $h \in \operatorname{Lip}_{v}^{\theta}(G)$. We choose $m$ such that it satisfies $q_{2}<m<p$. We conclude from Proposition 4, Lemmas 5, 11 and 12 that

$$
\begin{aligned}
\|[h, \tilde{\mathcal{R}}] f\|_{L^{p}(G)}^{p} \leq & \int_{G}\left|M_{\rho, \alpha}([h, \tilde{\mathcal{R}}] f)(x)\right|^{p} \mathrm{~d} x \\
\leq & C \int_{G}\left|M_{\rho, \beta}^{\sharp}([h, \tilde{\mathcal{R}}] f)(x)\right|^{p} \mathrm{~d} x \\
& +C \sum_{k}\left|Q_{k}\right|\left(\frac{1}{\left|Q_{k}\right|} \int_{2 Q_{k}}|[h, \tilde{\mathcal{R}}] f(x)| \mathrm{d} x\right)^{p} \\
\leq & C \int_{G}\left|M_{\rho, \beta}^{\sharp}([h, \tilde{\mathcal{R}}] f)(x)\right|^{p} \mathrm{~d} x \\
& +C\|h\|_{\operatorname{Lip}_{\nu}^{\theta}(G)}^{p} \sum_{k} \int_{2 Q_{k}}\left|\left\{M_{m v}\left(|f|^{m}\right)\right\}^{\frac{1}{m}}(x)\right|^{p} \mathrm{~d} x \\
\leq & C\|h\|_{\operatorname{Lip}_{\nu}^{\theta}(G)}\left\|\left(M_{m v}\left(|f|^{m}\right)\right)^{\frac{1}{m}}\right\|_{L^{p}(G)}^{p} \\
\leq & C\|h\|_{\operatorname{Lip}_{\nu}^{\theta}(G)}\|f\|_{L^{q}(G)}^{p},
\end{aligned}
$$

where $\frac{1}{p}=\frac{1}{q}-\frac{v}{D}$.

\section{Conclusions}

We prove the $L^{p} \rightarrow L^{q}$ boundedness for the commutator which is generated by the Riesz transform $\mathcal{R}$ and the function $h \in \operatorname{Lip}_{v}^{\theta}$. We generalize the corresponding results on the Euclidean space in [6] to the nilpotent Lie group, and they may have some applications in harmonic analysis and PDE on the Lie group.

\section{Acknowledgements}

Research supported by the National Natural Science Foundation of China (No. 11671031, 11471018), the Fundamental Research Funds for the Central Universities (No. FRF-BR-17-004B).

\section{Competing interests}

The authors declare that they have no competing interests.

Authors' contributions

All authors contributed equally to the writing of this paper. All authors read and approved the final manuscript.

\section{Publisher's Note}

Springer Nature remains neutral with regard to jurisdictional claims in published maps and institutional affiliations.

Received: 4 July 2017 Accepted: 6 December 2017 Published online: 19 December 2017

\section{References}

1. Varopoulos, NT, Saloff-Coste, L, Coulhon, T: Analysis and Geometry on Groups. Cambridge Univ. Press, Cambridge (1992)

2. Jiang, RJ, Jiang, XJ, Yang, DC: Maximal function characterizations of Hardy spaces associated with Schrödinger operators on nilpotent Lie groups. Rev. Mat. Complut. 24, 251-275 (2011)

3. Li, HQ: Estimations $L^{p}$ des opérateurs de Schrödinger sur les groupes nilpotents. J. Funct. Anal. 161, 152-218 (1999)

4. Coifman, R, Rochberg, R, Weiss, G: Factorization theorems for Hardy spaces in several variables. Ann. Math. 103, 611-635 (1976)

5. Janson, S: Mean oscillation and commutators of singular integral operators. Ark. Math. 16, 263-270 (1978)

6. Liu, Y, Sheng, JL: Some estimates for commutators of Riesz transforms associated with Schrödinger operators. J. Math. Anal. Appl. 419, 298-328 (2014)

7. Folland, GB: Subelliptic estimates and function spaces on nilpotent Lie groups. Ark. Mat. 13, 161-207 (1975) 
8. Folland, GB: Lipschitz classes and Poisson integrals on stratified groups. Stud. Math. 66, 37-55 (1979)

9. Yang, DaC, Zhou, Y: Localized Hardy spaces $H^{1}$ related to admissible functions on RD-spaces and applications to Schrödinger operators. Trans. Am. Math. Soc. 363, 1197-1239 (2011)

10. Liu, Y, Huang, JZ, Dong, JF: Commutators of Calderón-Zygmund operators related to admissible functions on spaces of homogeneous type and applications to Schrödinger operators. Sci. China Math. 56(9), 1895-1913 (2013)

11. Bernardis, A, Salinas, O: Two-weight norm inequalities for the fractional maximal operator on spaces of homogeneous type. Stud. Math. 108, 201-207 (1994)

Submit your manuscript to a SpringerOpen ${ }^{\circ}$ journal and benefit from:

- Convenient online submission

$\checkmark$ Rigorous peer review

Open access: articles freely available online

- High visibility within the field

- Retaining the copyright to your article

Submit your next manuscript at $\gg$ springeropen.com 